\title{
Alcances del turismo sostenible: un análisis cualitativo de las experiencias de dos comunidades en Ecuador
}

\section{Range of sustainable tourism: a qualitative analysis of the experiences of two communities in Ecuador}

Freddy Alejandro Barros Pinto $^{1 *}$

\author{
${ }^{1}$ Pontificia Universidad Católica del Ecuador, Facultad Ciencias Humanas, Carrera de Turismo. Avenida 12 de Octubre 1076 y \\ Vicente Ramón Roca. Quito, Ecuador. \\ 凶alejrexusid@yahoo.es \\ https://orcid.org/0000-0002-0111-9666
}

\begin{abstract}
Resumen
El presente estudio realizó un análisis cualitativo sobre el alcance del turismo sostenible generado por la ayuda técnica y financiera de la cooperación internacional y de organizaciones no gubernamentales (ONG) con sus proyectos en dos comunidades rurales en el Ecuador: la comunidad de Yunguilla y la comunidad de Oyacachi. Se efectuó una recopilación documental y un análisis del contenido sobre los informes técnicos de ejecución, con el fin de presentar las experiencias obtenidas a través de la adopción de la actividad turística sostenible en los territorios beneficiarios de estos proyectos. Se codificaron los datos precisos de los textos estudiados para realizar un análisis sistemático e interpretativo y exponer la narrativa sobre el alcance del turismo sostenible como una guía para el desarrollo en las comunidades objeto de esta investigación. Se concluye que la asistencia técnica y económica pueden ser las dos caras de la moneda del camino a la sostenibilidad.
\end{abstract}

Palabras clave: sostenibilidad, turismo sostenible, turismo alternativo, ecoturismo, desarrollo local.

\begin{abstract}
This research conducted a qualitative analysis on the scope of sustainable tourism caused by technical and financial support of international cooperation and NGOs projects in two rural communities in Ecuador: the community of Yunguilla and the community of Oyacachi. It was carried out a documentary compilation and content analysis of the technical financial execution reports, in order to present the experiences obtained through the adoption of sustainable tourism activities. The precise data of the studied texts were codified, then a systematic and interpretative analysis was made to expose the narrative on the scope of sustainable tourism as a guide for development in the communities that are the object of this research. The conclusion is that technical and economic assistance can be two sides of the same coin on the road to sustainability.
\end{abstract}

Keywords: sustainability, sustainable tourism, alternative tourism, ecotourism, local development. 


\section{Introducción}

El turismo alternativo asocia el tiempo libre, la recreación y la cultura con entornos de una extensa riqueza natural y cultural, convirtiéndose en una motivación para diversos turistas que aportan con divisas y apoyan la conservación del patrimonio natural y cultural en los países que apuestan por este tipo de turismo (Pons y López, 2006, p. 152). Según World Tourism Organization [UNWTO] (2019), el turismo en el mundo, al 2019, creció un $4 \%$ con relación al 2018. Sin embargo, para el primer trimestre del 2020 y debido a la pandemia del coronavirus (COVID-19), se presentó un decrecimiento de la actividad turística a nivel mundial (UNWTO, 2020a, pp. 7-8).

El mercado del turismo alternativo se incrementó un $25 \%$ entre el 2010 y el 2016, lo que representa más del $7 \%$ de la demanda turística global y mueve casi 123 billones de dólares anualmente (Leal Londoño, 2017). El turismo en el Ecuador es visto como una oportunidad de desarrollo económico para atenuar la pobreza e incluir a los sectores menos favorecidos (Caiza y Molina, 2012, p. 8), también se establece como una herramienta para la conservación de ecosistemas y sus servicios ambientales (Coppin, 1992; Pons y López, 2006); además de generar ingresos económicos, pretende disminuir los problemas socio ambientales como la tala de los bosques, la cacería y las actividades no compatibles con el ambiente (Hernández Pardo, 2011).

La adopción de esta modalidad turística proviene de la corriente de "desarrollo sostenible", discutida y acogida a nivel mundial desde los 70 $\mathrm{y}$ en un sinnúmero de cumbres mundiales en las décadas de los 80 y 90; proponiendo un modelo que implica el desarrollo de las localidades sin comprometer los recursos naturales para las generaciones presentes y futuras, y satisfaciendo las necesidades básicas de subsistencia (Bermeo Noboa, 2002; Reátegui Lozano, 2003).

Ecuador, al ser firmante y adherente de las cumbres de Río 1992 y Johannesburgo 2002, implementa la "Ley de Gestión Ambiental" el 30 de julio de 1999, con la que planifica el desarrollo sostenible desde una visión muy ambientalista con la adopción del ecoturismo (Jovanovic et al., 2018). Con base en lo descrito anteriormente, varios organismos interesados en apoyar la conservación del país comprometen recursos técnicos y económicos para alcanzar los objetivos de esos acuerdos.
Entre esos organismos están: la Unión Internacional para la Conservación de la Naturaleza [UICN], World Tourism Organization [UNWTO] u Organización Mundial de Turismo [OMT] [ambas pertenecientes a la Organización de las Naciones Unidas [ONU], la cooperación internacional con la Agencia de los Estados Unidos para el Desarrollo Internacional [USAID], Deutsche Gesellschaft für Technische Zusammenarbeit [GIZ], cooperación Belga, entre otras; y las ONG internacionales como The Nature Conservancy [TNC], World Wildlife Found [WWF] y Conservación Internacional [CI], quienes intervienen en el país con distintos programas y proyectos para procurar un cambio del uso irracional de los recursos naturales en el Ecuador (Rueda Flores, 2015, p. 55).

Con la ayuda técnica y financiera se espera incidir en el cambio del modelo productivo convencional para lograr una producción sostenible. De esta manera, la agricultura, la ganadería, el turismo y otras actividades productivas pueden impulsar la conservación de los recursos naturales (Azócar de Buglass, 1995b, p. 26).

La estrategia de aplicar este modelo productivo en los territorios con importantes espacios de biodiversidad y que lindan con áreas naturales protegidas, se enfoca en generar herramientas para apoyar los objetivos de conservación de dichos espacios y generar recursos económicos para las comunidades anfitrionas, sin afectar los ecosistemas y el patrimonio cultural (Pons y López, 2006, p. 162). Gracias a estos fondos y programas, en el Ecuador se han creado proyectos de turismo sostenible que apuestan por la conservación de sus recursos naturales y culturales y generan recursos económicos (Vargas Martínez et al., 2011, p. 409). Algunos de estos se han afianzado con el tiempo y han logrado consolidarse estableciendo un mercado específico, mientras que otros han sucumbido en el intento.

Conforme a las intervenciones y a la cooperación internacional y de ONG nacionales e internacionales establecidas en el país por casi cuatro décadas, queda la interrogante: ¿Cuáles han sido los alcances de la aplicación del turismo sostenible en los territorios rurales que albergan una alta biodiversidad y cultura? Varios de estos proyectos han sido avalados por el Ministerio del Ambiente del Ecuador y fueron aceptados por varias comunidades en distintos cantones a nivel nacional. Sin embargo, todos estos informes descansan en los expedientes que dan fe de su ejecución, sin ser sistematizados, de tal 
manera que pueden dar a entender las dinámicas y aprendizajes obtenidos de estos procesos en los territorios beneficiarios.

El objeto de este estudio es analizar de forma cualitativa el alcance que tuvo la ejecución de los proyectos de desarrollo de turismo sostenible en dos comunidades del Ecuador, que estimularon a estos territorios a cambiar su modelo productivo convencional, a un modelo productivo sostenible, a través de la ayuda técnica y financiera de la cooperación internacional y nacional.

El análisis se realizó mediante la recopilación de los documentos técnicos resultado de las experiencias implementadas en las comunidades y se sistematizó con la metodología de "análisis de contenido" para especificar las propiedades de los procesos de desarrollo. De esta manera, se estableció una interpretación de los resultados obtenidos sobre la aplicación de turismo sostenible en las zonas de estudio.

El concepto de desarrollo, hasta mediados del siglo XX, ha sido definido como el crecimiento económico generado por los excedentes monetarios resultantes de la producción y comercialización incesante de la industria, el comercio y empresas. El impacto ambiental ha generado importantes cambios en el ambiente debido a la sobreexplotación de los recursos naturales en el planeta y la inadecuada gestión de sus residuos; estableciéndose como un modelo económico insostenible en el mundo (Reátegui Lozano, 2003, p. 68).

Varios países se han preocupado por el deterioro ambiental producto del crecimiento de la industrialización que genera contaminación y es causante del actual cambio climático en el mundo. La ONU encabeza varios espacios de debate y acuerdos desde 1972, 1983, 1992 y el año 2000, donde se enfatiza la necesidad de generar un nuevo modelo de desarrollo que integre a los seres humanos y la naturaleza, garantizando un manejo adecuado de los recursos naturales y un desarrollo sostenible de la humanidad (Bermeo Noboa, 2002, p. 2; Strydom et al., 2019, p. 5).

Por lo expuesto anteriormente, la ONU (2020) define como desarrollo sostenible:

El desarrollo sostenible constituye un proceso que pretende la satisfacción de las necesidades actuales permanentemente, sin comprometer la satisfacción de las necesidades futuras de las actuales generaciones y de las que vendrán, es decir, que no agota ni desperdicia los recursos naturales y no lesiona innecesariamente al ambiente ni a los seres humanos. (p. 4)

Reátegui Lozano (2003) destaca que "el desarrollo se lo entiende como un proceso de cambios cualitativos y de transformaciones de las estructuras económicas, sociales y políticas, en armonía con los sistemas naturales". Entendiéndose como un modelo de desarrollo que propone una amplia gama de posibilidades para la población, enfocándose en tres pilares fundamentales: social, ecológico o ambiental y económico (pp. 69-70).

En 1991, la Asociación Internacional de Expertos Científicos en Turismo establece el término "turismo sostenible" como un concepto que equilibra los beneficios sociales, económicos y ecológicos; este precepto no implica impactos negativos sobre los tres pilares fundamentales de desarrollo sostenible (López, 2005, p. 336; Sánchez Valdés y Vargas Martínez, 2015, p. 348). La UNWTO (2020b) lo concibe como una actividad productiva que gestiona eficientemente los recursos naturales y culturales, satisfaciendo las necesidades económicas, sociales y estéticas de las poblaciones locales y los turistas; preservando los componentes ecológicos, culturales y sociales del territorio que lo aplica.

En articulación con el término, y para ser congruente con el marco epistemológico del desarrollo sostenible, la UNWTO (2020b) define turismo sostenible como:

El turismo que tiene plenamente en cuenta las repercusiones actuales y futuras, económicas, sociales y medioambientales para satisfacer las necesidades de los visitantes, de la industria, del entorno y de las comunidades anfitrionas. (p. 1)

Por su concepto, el turismo sostenible reduce la degradación del ambiente generado por los impactos de la actividad turística sobre los recursos ecológicos y culturales, a través de la planificación y participación de la población local y aplicando una visión a largo plazo, motivando el consumo sostenible de los recursos presentes en el territorio a partir de los tres pilares de la sostenibilidad: ecológico-ambiental, social-cultural y económico (Jovanovic et al., 2018; Kapera, 2018). 
La actividad turística se adapta en este marco conceptual con el objetivo de lograr el bienestar de las poblaciones locales anfitrionas, de los operadores turísticos y de los turistas en relación con el destino turístico, sin dejar de lado el crecimiento económico característico de esta actividad y buscando crear conciencia en cuanto a la necesidad de conservar el patrimonio cultural y ambiental en beneficio de la población mundial (Vargas Martínez et al., 2011, pp. 409-410).

Los beneficios que genera el turismo sostenible han despertado el interés de los gobiernos de países en desarrollo, ya que es una de las actividades económicas compatibles con la conservación del ambiente y su calidad en los núcleos turísticos, además de contribuir al desarrollo económico y al alivio de la pobreza, con su capacidad de generar divisas, favoreciendo la inversión privada; el desarrollo de empresas locales, la creación de empleo y fuentes de ingresos alternativas, vistos como incentivos para dejar de lado la explotación insostenible de los recursos naturales (Altés, 2006).

En América Latina, el turismo sostenible es visto como una alternativa para contribuir a las acciones en contra del cambio climático e impulsar ingresos económicos. Diversos países en la región adoptan esta modalidad como una herramienta que apoya las acciones de conservación, rechaza el modelo de turismo de masas y le apuesta a la generación de experiencias auténticas a través de la oferta de productos y servicios turísticos de calidad, compatibles con el ambiente y la cultura anfitriona (Coppin, 1992, p. 9; Sundström, 2003, p. 10).

Con base en estos principios, y conforme al crecimiento de los segmentos de mercado que buscan productos que los acerquen con entornos conservados de una forma más directa y activa, que demuestren responsabilidad en su operación permitiéndoles valorar y respetar las características propias de los escenarios naturales y socioculturales de las poblaciones locales en los destinos visitados, se establece el concepto de turismo alternativo: "Modalidad turística que permite el contacto directo del turista con las comunidades y su entorno natural y cultural, participando en su conservación y aporte a su desarrollo" (Secretaría de Turismo [SECTUR], 2004, p. 22; Torres Fernández et al., 2013, p. 129).

El turismo alternativo agrupa tres modalidades: ecoturismo, turismo de deportes y aventura, y turismo rural. Cada uno con sus propios conceptos, características y actividades que permiten aplicar los principios de sostenibilidad, beneficiando no solo a las comunidades anfitrionas sino también a los turistas que optan por estas modalidades turísticas.

Costa Rica, Perú y Ecuador, desde la década de los 80 , han adoptado la modalidad de ecoturismo como una herramienta que apoya la filosofía del desarrollo sostenible, ya que se enmarca en el cuidado de la diversidad de plantas y animales que habitan en los bosques nativos, que en estos países todavía cubren una importante superficie de sus territorios. La adopción del ecoturismo se hace con el objeto de evitar la explotación y la devastación de los bosques con fines de extracción de materias primas y yacimientos de varios tipos, así como la extracción de petróleo (Strydom et al., 2019; Sundström, 2003), favoreciendo la conservación ambiental y la generación de estrategias para involucrar la participación social; logrando la valorización de los recursos y propiciando la concientización tanto de la población local, así como de los turistas, sobre la importancia de los servicios ecosistémicos que proporcionan sus territorios. El resultado es la generación de beneficios económicos mediante la creación de alternativas de trabajo y actividades económicas complementarias a las actividades agrícolas y pecuarias en las zonas de alta biodiversidad, que son presionadas por actividades extractivistas y malas prácticas productivas (Manuel Aragón, 2014, pp. 13-14).

Esta actividad productiva en los últimos años ha sido aplicada como un producto muy atractivo a ofertar, ya que su esencia atrae a varios turistas que tienen una conciencia sobre los problemas ambientales, económicos y sociales del mundo, quienes buscan productos que apoyen al desarrollo socioeconómico de las comunidades locales, a la adecuada gestión, su conservación y respeto de los recursos naturales, culturales-patrimoniales locales; aplicando políticas y acciones en el marco de un turismo sostenible (Orgaz-Agüera \& Cañero Morales, 2017, pp. 100-101).

Las poblaciones que se han visto involucradas con la valoración, planificación y ejecución de proyectos de turismo sostenible en sus territorios logran establecer una visión de desarrollo desde sus propias experiencias y expectativas, logrando mantener su estilo de vida en armonía con su entorno, sin dejar de recibir réditos económicos gracias a la aplicación de buenas prácticas de sus actividades productivas; a esta capacidad se la conoce como desarrollo local (Boisier, 2001, p. 7). 
Desde este enfoque, el turismo sostenible es gestionado por la comunidad, involucrando a sus pobladores de forma participativa en la gestión y uso sostenible de sus recursos. Esta gestión promueve, en los actores locales, las habilidades de asociatividad, trabajo en red, trabajo en género; cumpliendo así con los objetivos planteados desde su propia visión comunitaria, adaptando y optimizando sus procesos para mejorar su condición de vida (Carrillo García et al., 2017, p. 565).

Las comunidades locales planifican esta actividad con base en las capacidades reales de sus territorios, tanto de sus atractivos naturales y culturales, así como en la integración de los servicios básicos y la planta turística existentes, vinculando la participación de la comunidad local a través de la gestión y operación del producto turístico y la facilitación y atención del turista. Sin embargo, este tipo de desarrollo también es criticado por su alta dependencia con las ONG, así como por las escasas capacidades de la población local para la generación de una adecuada visión de desarrollo, poniendo en riesgo el alcance de los ámbitos socioeconómico, cultural y ambiental de sus zonas (Orgaz-Agüera \& Cañero Morales, 2015, pp. 102-103).

Prieto (2011) establece dos momentos importantes en el desarrollo turístico del Ecuador. En el periodo presidencial de Galo Plaza, existió mayor énfasis en la promoción turística del país como una estrategia de desarrollo económica, con la creación de una oficina adscrita a la Presidencia de la República orientada a publicitar a Otavalo y Galápagos. Por otro lado, se promovió el establecimiento de la infraestructura hotelera y la creación de la agencia de viajes Metropolitan Touring. También se menciona que el país dio un giro hacia el turismo sostenible abriendo las puertas a ONG y cooperación internacional, con el objeto de implementar el ecoturismo a través de las intervenciones económicas y la asistencia técnica en comunidades y gobiernos locales, con un enfoque de conservación de los lugares con una alta biodiversidad y con el fin de disminuir las fuentes de presión hacia los recursos naturales. En este periodo, el país no solo mostraba como oferta turística los destinos de Quito, Otavalo y Galápagos, sino que también se abrían nuevos destinos en la Amazonía, Andes y la Costa ecuatoriana (Prieto, 2011).

Arrueta (1994) menciona que el ecoturismo se inicia en la región amazónica en la década de los 90 con la creación del Instituto Ecuatoriano Forestal y de Áreas Naturales y Vida Silvestre (INEFAN) - Re- gistro Oficial No. 27, de 16 de septiembre de 1992-, adscrito al Ministerio de Agricultura y Ganadería de esa época; cumpliendo con lo dispuesto en la Ley Forestal y de conservación de Áreas Naturales y Vida Silvestre, el Reglamento General de aplicación y demás disposiciones legales y reglamentarias referente el recurso forestal, a las áreas naturales y a la vida silvestre. El INEFAN, además de gestionar las áreas protegidas estatales, debía coordinar el desarrollo turístico en las áreas protegidas con el consejo de turismo CETUR, en el marco de los acuerdos internacionales para la conservación de la naturaleza y sostenibilidad. Sin embargo, como se menciona en este estudio, “en la década de los 70's en las cuencas de los ríos Aguarico, Napo y Cuyabeno ya se realizaba actividades turísticas y paseos de aventura por la selva". (Arrueta, 1994). También menciona que desde la creación de la Reserva Faunística Cuyabeno, en 1979, el ingreso de turistas aumentó por las operaciones de ecoturismo realizados por Neotropic Tourism (hoy en día conocido como Cuyabeno Lodge), aprovechando las características físico-geográficas, ecológicas e hidrográficas de la reserva.

Otras experiencias similares en el Ecuador, bajo el enfoque de turismo sostenible, son recopilados por Azócar de Buglass et al. (1995a) en el libro Ecoturismo en el Ecuador. Trayectorias y desafios, en el que se exponen experiencias comunitarias como: La comunidad de Agua Blanca - Parque Nacional Machalilla; comunidad Capirona - provincia del Napo; comunidad Cofán de Zábalo - provincia de Sucumbíos, así como las experiencias de empresas privadas: CEMECOTUR - TRANSTURI "Flotel Orellana", Hostería Alándaluz; y de proyectos ejecutados por ONG: Fundación Antisana - "Cóndor del Antisana", Fundación Natura - "Bosque Protector Pasochoa", Fundación Jatun Sacha - "Estación Biológica", Fundación Maquipucuna - "Ecoturismo en Bosque Nublado".

\section{Materiales y métodos}

La presente investigación tiene un enfoque cualitativo con un alcance exploratorio basado en el diseño de la teoría fundamentada. La metodología escogida para este trabajo se basa en el análisis del contenido (Aigeneren, 1999; Andréu Abela, 2000; Espín López, 2002), estableciéndose como una técnica de investigación realizada de manera objetiva y sistemática sobre los informes, memorias y reportes técnicos de las actividades y metodologías aplicadas en las intervenciones de los proyectos de cooperación de organismos 
internacionales para el apoyo al desarrollo del turismo sostenible en las zonas de estudio.

Como método se desarrolló, en primera instancia, la recolección y selección de los documentos a analizar, identificando especialmente aquellos que están vinculados con el desarrollo de la actividad turística sostenible en las comunidades de Yunguilla y Oyacachi respectivamente. Una vez escogida la fuente de información, se procedió a analizar y formular el tipo de objetivo que se persigue trabajándolo en dos fases: la primera corresponde a la recolección de la literatura relacionada con el tema de estudio, con la que se va a construir el marco teórico sobre las características y alcances del turismo sostenible, y la segunda fase consiste en desarrollar el objeto de estudio.

En un segundo momento se procedió a elaborar los indicadores o unidades de análisis. En este sentido, como lo menciona Andréu Abela (2000, p. 16), se procedió a codificar el material recolectado y distribuirlo en cada categoría de estudio. Para aque1lo, se tomó en consideración el objeto o referente a analizar "el alcance del turismo sostenible en las zonas de estudio", aplicando como herramienta el método de "frecuencia o el porcentaje de aparición" con el fin de extraer los datos precisos de los textos a estudiar; por lo dicho se logra construir el siguiente sistema de categorías:
a. Ubicación y caracterización de las zonas de estudio
b. Antecedentes socio económico de las localida- des a estudiar
c. Activación de turismo sostenible
d. Estructura de la organización comunitaria
e. Desarrollo económico a través del turismo
f. Estructura de la oferta y demanda turística
g. Resultados económicos por turismo
h. Desarrollo social
i. Conservación de su entorno natural

Conforme los datos cualitativos conseguidos en los recursos bibliográficos recolectados y categorizados, se procedió con la fase de análisis a través del tratamiento del texto con fichas de contenido. Cabe recalcar que los datos económicos de la comunidad de Yunguilla fueron más precisos, de acuerdo al estudio económico realizado en el territorio, por lo que se presenta una tabla que interpreta los ingresos y egresos generados por la actividad turística y el gráfico de barras que permite visibilizar esos ingresos en un periodo de 7 años, lo que no ocurrió con los datos obtenidos en los informes de la comunidad de Oyacachi. Por último, se procedió a la sistematización e interpretación de los resultados obtenidos según las categorías a estudiar, permitiendo exponer de forma narrativa el alcance del turismo sostenible como mecanismo de desarrollo en las dos comunidades objeto de estudio.

\section{Resultados}

\subsection{Alcances del turismo sostenible en la comuni- dad de Yunguilla}

La comunidad de Yunguilla, ubicada en la parroquia de Calacalí del Distrito Metropolitano de Quito, linda con la Reserva Geobotánica Pululahua área nacional protegida del Ecuador; se encuentra a una altitud aproximada de $2.650 \mathrm{~m} \mathrm{~s}$. n. m. En la actualidad, este territorio forma parte del Subsistema Metropolitano de Áreas Protegidas del Municipio del DMQ, bajo la categoría de Área de Conservación y Uso Sustentable Yunguilla, declarada bajo Ordenanza Municipal N. ${ }^{\circ} 049$ de 11 de julio de 2013 (Carrera et al., 2016).

Yunguilla está conformada por 56 familias; con una población aproximada de 220 habitantes que apostaron a la conservación desde 1995 con el apoyo de la Fundación Maquipucuna, quienes realizaron un estudio socioeconómico de la cuenca alta del río Guayllabamba y observaron la pérdida de bosque ocasionada por la falta de alternativas productivas en la zona (Maquipucuna, 2019b), lo que acarreó a la comunidad de Yunguilla y comunidades vecinas a explotar el bosque desmesuradamente con actividades tradicionales como la agricultura, ganadería y la extracción de madera para carbón, agotando los recursos existentes (Neudel, 2015, p. 53).

Al ver que se generaban pocos recursos económicos por las malas prácticas y que los bosques disminuían a medida que el tiempo pasaba, Yunguilla decide participar en algunos proyectos generados por la Fundación Maquipucuna, con el apoyo de Rain Forest Alliance y financiados a través de los fondos del Programa de Pequeñas Donaciones (PPD) del Programa de las Naciones Unidas para el Desarrollo (Neudel, 2015). La ejecución del proyecto ecoturístico se enmarcó en aprovechar la belleza paisajística del Bosque Nublado donde se asienta la comunidad, con el objetivo de mejorar la calidad de vida de sus pobladores mediante el aprovechamiento y manejo adecuado de los recursos naturales existentes en la zona. 
El proyecto se enfocó en brindar un acercamiento al turista con la biodiversidad existente en la zona y de esta manera aprovechar los caminos ancestrales existentes, también llamados culuncos ${ }^{1}$. Estos caminos ancestrales son utilizados para senderismo y, sobre todo, para demostrar el modo de vida y las expresiones culturales de la gente que vive en esta zona (Maquipucuna, 2019a).

Bajo estos parámetros, un grupo de jóvenes se organizó y conformó el grupo básico de operaciones turísticas de la comunidad. El proyecto determinó realizar un ciclo de capacitaciones para formar guías locales con 16 personas de la comunidad entre hombres y mujeres, también se capacitó a las familias en nutrición e higiene con el fin de mejorar el valor nutricional y calidad de su oferta gastronómica, con el fin de brindar servicios de alimentación.

De la misma manera, fue necesario capacitarlos en la administración y manejo contable de la operación turística y, por último, se capacitó a miembros de la comunidad en el tema de construcción alternativa de infraestructura y edificaciones con materiales acordes con el ambiente, a fin de ir adaptando facilidades e infraestructura turística bajo los principios de sostenibilidad (Maquipucuna, 2019a, 2019b; Neudel, 2015).

El modelo de gestión de la actividad turística, así como de otros procesos productivos comunitarios, como la elaboración de artesanías, papel reciclado, mermeladas y productos derivados de la producción de leche, lo gestiona la Corporación Microempresarial Yunguilla, entidad conformada por dos grupos de la misma comunidad, desde 1999 hasta la actualidad (Neudel, 2015).

El 5 de junio del 2000, el Ministerio de Relaciones Laborales, mediante Acuerdo Ministerial N. ${ }^{\circ}$ 063, otorgó la personería jurídica a la organización comunitaria llamada Corporación Microempresarial Yunguilla, vinculando en su estructura funcional a los miembros que obtuvieron destrezas y habilidades en la administración, planificación, gestión de proyectos y la administración de los recursos financieros (Cárdenas Toscano, 2016, pp. 49-50; Acuerdo Ministerial $\mathrm{N}^{\circ} 063$ ). La corporación está compuesta por personas escogidas en una asamblea comunitaria, quienes ocuparán un puesto de coordinación para el desarrollo de cada unidad productiva, lo recaudado se destina a pagar los sueldos de las personas que participan en las prestaciones de servicios y actividades de turismo comunitario, así como al pago de la producción agrícola y pecuaria de la comunidad. Los excedentes económicos resultantes de la comercialización de los productos los utilizan para mejoras en la comunidad.

Otro de los componentes del proyecto de ecoturismo implementado en ese tiempo, fue el énfasis en la adecuación de la infraestructura y equipamiento turístico en la comunidad. Se adecuó la casa hacienda de Tahuallullo para el hospedaje de 10 pax y el servicio de alimentación para 40 pax, se habilitó un área de camping con un aforo para 30 pax y se crearon cuatro rutas dentro de la comunidad y el bosque nublado. Conforme fue desarrollada la operación turística en la comunidad, y a medida que se fueron especializando en las necesidades de la demanda del producto ecoturístico, la comunidad fue adaptando sus viviendas para recibir turistas en las casas de cada familia interesada en participar en esta actividad productiva y de esta manera generar un ingreso económico para las familias de la comunidad (Corporación Microempresarial Yunguilla, 2020).

El último componente del proyecto es la promoción, para lo cual se creó la página web con la que se expone de manera directa los paquetes turísticos ofertados por la comunidad, así como su historia y otros proyectos realizados. Se ejecutó un plan de marketing para la operación del producto de ecoturismo de Yunguilla; este plan estipuló la elaboración de folletos y trípticos de promoción turística.

En la actualidad, el producto de ecoturismo pasó a ser un producto de turismo comunitario con enfoque de sostenibilidad. Además de realizar actividades inherentes a esta modalidad, los viajeros tienen la posibilidad de convivir con las familias de la comunidad, realizando actividades típicas y tradicionales conforme a su oferta actual; el segmento de mercado de Yunguilla, en su mayor proporción, son extranjeros de Holanda, Suecia e Inglaterra. Los fines de semana y feriados, el mercado es local y se origina en la ciudad de Quito, la visita a la comunidad la realizan para disfrutar de caminatas, camping y del nuevo restaurante comunitario, creado con recursos de la Corporación Yunguilla, la mano de obra de sus habitantes y con aportes económicos del Fondo Ambiental del GAD - DMQ (Secretaría de Ambiente del GAD DMQ, 2015), Quito Turismo,

${ }^{1}$ De acuerdo con Jara Chávez (2006) "Culuncos son la red vial de la cultura Yumbo que fue trazado por todo su territorio". 
Ministerio de Turismo del Ecuador y el GAD de Pichincha, obtenidos de la gestión de la corporación.

Yunguilla se dedica al desarrollo sostenible y a la conservación de sus recursos, apostándole al turismo sostenible; la Corporación decidió realizar un análisis económico y la evaluación del cumplimiento del plan de manejo del ACUS Yunguilla del periodo 2012-2016; en el marco del proyecto "Fortalecimiento a la gestión comunitaria para la conservación del ACUS Yunguilla-Santa Lucía”, ejecutado por la Corporación Yunguilla y la Cooperativa Santa Lucía con el apoyo de Critical Ecosystem Partnership Fund y el respaldo del
Municipio del DMQ a través de la Secretaría de Ambiente (Corporación Microempresarial Yunguilla, 2018).

La economista Consuelo Rojas Almeida realizó el análisis de los ingresos y gastos generados en las diversas fuentes de financiamiento de Yunguilla, por lo que recopiló los datos generados de las actividades de turismo (ingresos y gastos económicos) en un periodo de seis años entre el 2010 y 2016. Con estos datos realizó un análisis de los ingresos versus los gastos e identificó importantes rubros como utilidades obtenidas en cada año (Tabla 1).

Tabla 1. Registro de ingresos y egresos por turismo de Yunguilla

\begin{tabular}{cccc}
\hline \multicolumn{4}{c}{ RUBRO PRODUCTIVO: TURISMO } \\
\hline Años & Ingreso & Gasto & Utilidad \\
2010 & $36.564,94$ & $25.359,58$ & $11.205,36$ \\
2011 & $30.984,57$ & $26.746,62$ & $4.237,95$ \\
2012 & $40.468,38$ & $35.716,47$ & $4.751,91$ \\
2013 & $54.623,30$ & $46.309,10$ & $8.314,20$ \\
2014 & $69.348,66$ & $55.687,32$ & $13.661,34$ \\
2015 & $86.629,58$ & $72.189,31$ & $14.440,27$ \\
2016 & $101.816,72$ & $81.845,41$ & $19.971,31$ \\
\hline
\end{tabular}

Fuente: Corporación Microempresarial Yunguilla. (2018).

Se observa que los ingresos generados por la oferta de los servicios y productos turísticos de la comunidad de Yunguilla, menos los gastos de operación turística de la Corporación Yun- guilla, permitieron obtener una utilidad promedio de US\$ 10.940,33 en cada año (Figura 1), los mismos que fueron reinvertidos en proyectos de la comunidad.

Figura 1. Análisis de ingresos, gastos y utilidades periodo 2010 - 2016 (Corporación Microempresarial Yunguilla., 2018).

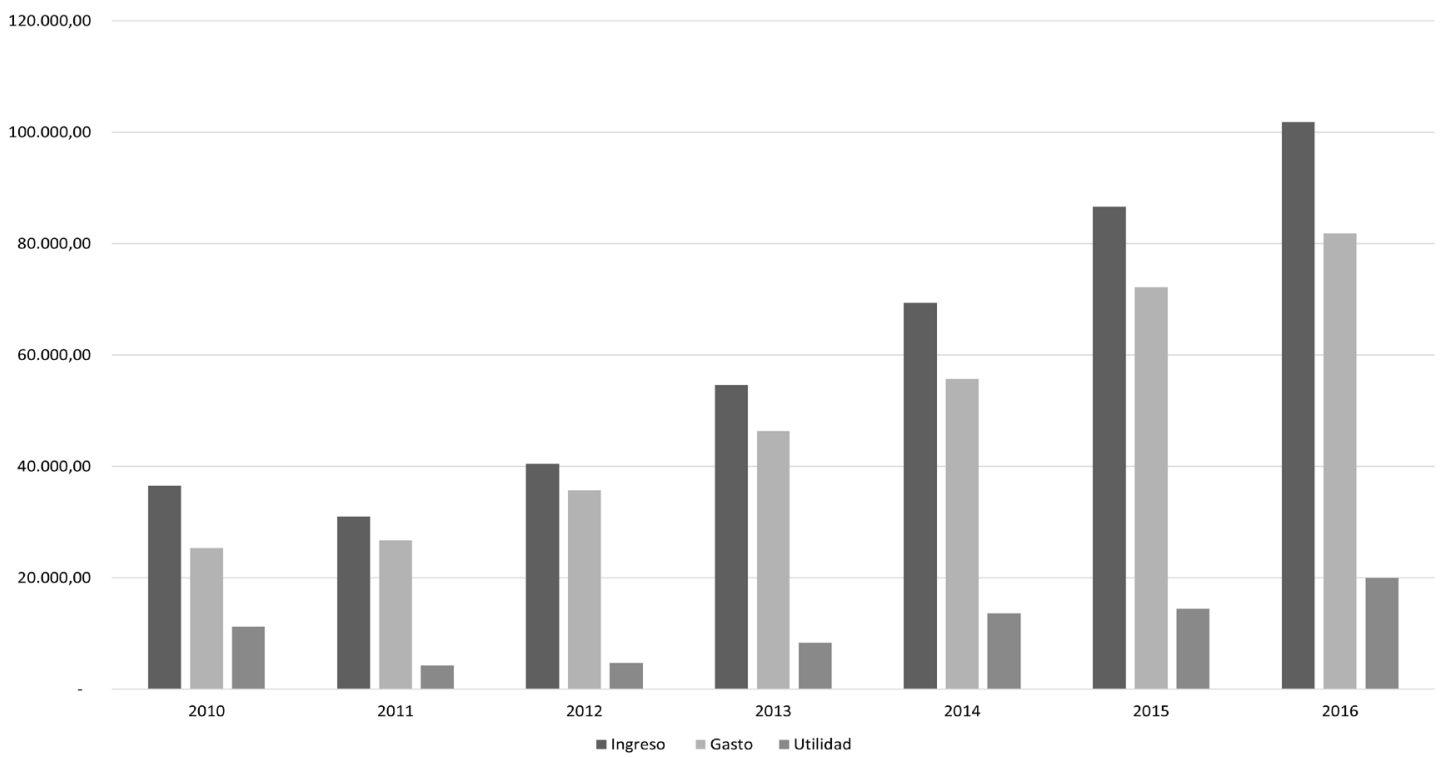


Los réditos económicos obtenidos, sumados a su visión de ser una comunidad sostenible, motivan a los miembros de la comunidad a seguir un desarrollo comunitario basado en el aprovechamiento de sus recursos naturales y culturales a través del trabajo comunitario, reinvirtiendo las utilidades generadas en beneficio de la comunidad (Orgaz-Agüera \& Cañero Morales, 2017).

La comunidad de Yunguilla, al dejar de talar sus bosques e implementar actividades productivas sostenibles, entre ellas el turismo sostenible, mantiene un importante incremento de cobertura vegetal en regeneración, así como el retorno de fauna silvestre a sus bosques. Se puede observar especialmente el compromiso de la gente en mantener un estilo de vida amigable con su entorno (Corporación Microempresarial Yunguilla, 2018).

\subsection{Alcances del turismo sostenible en la comunidad de Oyacachi}

Oyacachi es una parroquia del cantón El Chaco de la provincia de Napo. La comunidad kichwa de Oyacachi o "cara de sal" (en su traducción al español) (Lasso, 2015), está situada en las laderas orientales de la Cordillera de los Andes, a $45 \mathrm{~km}$ del Distrito Metropolitano de Quito, ubicada dentro del Parque Nacional Cayambe-Coca. El territorio de Oyacachi va desde los $1.880 \mathrm{~m} \mathrm{s.} \mathrm{n.} \mathrm{m.} \mathrm{a} \mathrm{los} 4.800 \mathrm{~m}$ s. n. m., las características de su paisaje, así como la conformación de sus suelos, hacen que el territorio sea un lugar propicio para la agricultura y el turismo (Centro para la Investigación de la Diversidad Cultural y Biológica de los Bosques Pluviales Andinos [DIVA], 2000).

La comunidad mantiene un territorio de 44.500 ha, está conformada aproximadamente por 620 habitantes (Instituto Nacional de Estadística y Censos del Ecuador, 2019), sus principales actividades económicas son la agricultura, la ganadería, la piscicultura, artesanía (tallado de madera) y el ecoturismo (Manuel Aragón, 2014; Lasso, 2015). En 1997, en el centro poblado se construyó el complejo termal "Centro Termal Recreativo Oyacachi" y la construcción de cabañas para la recepción de turistas cercano al complejo termal. Esta planta turística fue edificada con el apoyo del proyecto "Sustainable Use of Biological Resources - SUBIR II", dirigida por la Fundación Antisana (Centro para la Investigación de la Diversidad Cultural y Biológica de los Bosques Pluviales Andinos [DIVA], 2000, pp. 57-104).
Con la dotación de esta infraestructura, la comunidad ha desarrollado la actividad turística de manera espontánea. Ésta se enfoca en la modalidad de turismo en aguas termales; el ecoturismo apoyado en la belleza escénica del paisaje y la conservación de los ecosistemas; y el turismo cultural, basado en sus valores culturales (Alex C. Walker Foundation et al., 2007, p. 21). La mano de obra de la comunidad se la presentó como contrapartida para la ejecución del proyecto y lo gestionó el Comité de Ecoturismo y el Cabildo parroquial (Flores, 2007, p. 12).

El Gobierno Autónomo Descentralizado (GAD) parroquial maneja la infraestructura turística de Oyacachi; antes, la entidad para la gobernanza y desarrollo turístico de la parroquia fue conformada por el cabildo, constituido por un presidente, vicepresidente, tesorero, secretario, síndico y los vocales dedicados a desarrollar las actividades productivas agropecuarias, de ecoturismo, artesanías, y una caja de ahorro y crédito (Fondo para la protección del Agua [FONAG], 2018, p. 24). La administración del complejo y de toda la infraestructura turística estuvo a cargo del cabildo de Oyacachi como negocio comunitario y la rendición de cuentas por gastos y ganancias se las presentaban en asamblea comunitaria (Bustamante, 2007, pp. 5-6).

Alex C. Walker Foundation et al. (2007) exponen: "En el año 2000 la comunidad de Oyacachi elaboró su plan de manejo con el fin de contar con una herramienta que les permitiera impulsar el desarrollo en la comunidad sin causar daños a los recursos naturales" (p. 22). En ese marco, la comunidad y el cabildo, en asamblea, apoyaron la implementación de un proyecto de ecoturismo para la creación del Comité de Ecoturismo.

También apoyaron el Ministerio del Ambiente del Ecuador, Ecociencia, The Nature Conservancy, entre otras. El Comité de Ecoturismo se encargó de temas como la planificación del proyecto, el enlace de todas las personas involucradas en el ecoturismo en la comunidad, la coordinación con las organizaciones externas y el seguimiento de la actividad ecoturística, con el fin de impulsar un proyecto que contribuya al desarrollo de la comunidad y la conservación de la biodiversidad existente en su territorio (Comité de Ecoturismo Oyacachi y EcoCiencia, 2007).

En 2001, el Programa Parques en Peligro (PEP) en la Bioreserva del Cóndor, financiado por The Nature Conservancy y Agencia de los Estados Unidos para el Desarrollo, apoyó el proyecto de ecoturismo 
con líneas concretas de intervención: análisis de factibilidad, compuesto por un estudio de mercado, un estudio financiero, un diagnóstico social, y un estudio de capacidad de carga de nuevos sitios para senderismo en Oyacachi (Alex C. Walker Foundation et al., 2007, pp. 8-9), planificación turística-arquitectónica y un programa de fortalecimiento y capacitación para los actores turísticos del territorio (Flores, 2007).

Con la dotación de infraestructura y servicios turísticos para la atención de turistas, Ecociencia con el Programa Parques en Peligro, y en coordinación con el Comité, deciden instalar una oficina de turismo para brindar información a los visitantes, a través de materiales promocionales como trípticos con referencia a los servicios de ecoturismo y de educación ambiental, enfatizando el conflicto oso-ganado. También se diseñó un paseo turístico para Oyacachi (Carvalho \& Rodríguez, 2006; Flores, 2007).

El mismo año, la Fundación Esquel, en el marco del proyecto, "Apoyo a iniciativas de turismo comunitario", formuló la planificación estratégica del Comité de Ecoturismo, e impartió cursos de capacitación con carácter técnico: cocina, guianza y buenas prácticas ambientales. Mientras que el Programa Parques en Peligro desarrolló una capacitación dirigida a los guías del Comité de Ecoturismo llamado "Entrenamiento en acción" para el establecimiento de nuevas rutas para senderismo (Papallacta-Oyacachi, Oyacachi-El Chaco) (Flores, 2007, p. 12).

El complejo turístico de Oyacachi, conformado por cinco cabañas, tres piscinas medianas y dos pequeñas, generó un promedio de utilidades de US\$ $13.960,00$ entre los años 2001 y 2005, por el cobro de ingresos al centro termal (Bustamante, 2007). Como lo establece Carvalho \& Rodríguez (2006), el principal mercado que consume este producto "está conformado por campesinos de clase media-baja que viven en las comunidades vecinas de Cangahua y Cayambe".

Después de casi 13 años, la actividad turística en el territorio se ha visto limitada por una débil organización comunitaria, ya que las operaciones turísticas se basan únicamente en la atención de bañistas en el centro termal. Los ingresos por turismo se limitan al cobro de la entrada al balneario, y los escasos ingresos económicos recibidos por esta actividad son direccionados por el GAD a la atención de obras públicas, dejando de lado la inversión para el desarrollo sostenible de la comunidad y la conservación de los ecosistemas en Oyacachi (Lasso, 2015).
En la actualidad, los esfuerzos de cambio de actividades productivas convencionales a sostenibles no han sido estudiados, no se puede definir si existe un cambio cualitativo y cuantitativo sobre la conservación de los ecosistemas a través de la aplicación del turismo sostenible en Oyacachi.

\section{Discusión}

Esta investigación expone las experiencias obtenidas de las comunidades de Yunguilla y de Oyacachi en los procesos generados por la ejecución de la ayuda técnica y económica para el desarrollo sostenible en sus territorios. Estas experiencias no están condicionadas únicamente por la ejecución de la asistencia técnica y financiera para la promoción de la conservación de los recursos naturales y la obtención de rentabilidad económica y social en la operación de sus productos turísticos, sino también por el interés, apoyo y capacidades de las comunidades para optar por un modelo de desarrollo alternativo.

Estos procesos se enmarcaron en el concepto de desarrollo sostenible (ONU, 1992), instituyéndose para las ONG como una herramienta que permita disminuir el detrimento de la cobertura vegetal, que genere ingresos económicos y que sea participativa a nivel local. Desde esta base se generó un cambio en las comunidades beneficiarias en su orientación y modo productivo tradicional hacia un estilo de vida sostenible con su entorno; vinculando a sus pobladores en la obtención de beneficios en los servicios ecosistémicos, rentabilidad productiva y un desarrollo socio cultural a través de una adecuada planificación y gestión de su territorio a largo plazo, en favor de las actuales y futuras generaciones (Cruz Albornoz, 1993; UNWTO, 2020b).

Los estudios sobre turismo sostenible se enfocan principalmente en el uso de los recursos naturales, tratando muy poco el tema sociocultural como uno de los pilares de la sostenibilidad, que en las últimas décadas empieza a ser tomado en cuenta para su análisis y discusión (Vargas Martínez et al., 2011). Sin embargo, los alcances de este estudio identifican las particularidades de cada comunidad, examinado sus capacidades intrínsecas en el empoderamiento, liderazgo y la puesta en práctica de las lecciones aprendidas de los procesos en que cada una de ellas han participado (Neudel, 2015).

Estas experiencias están alineadas en los principios de sostenibilidad, asegurando el bienestar de 
la población local y su educación, beneficiando a sus microempresas, activando la participación local y contribuyendo a la conservación de su patrimonio natural y cultural (Jovanovic et al., 2018; Sánchez Valdés \& Vargas Martínez, 2015). Aunque la comunidad de Oyacachi no logra afianzar su proceso de desarrollo local como lo ha hecho la comunidad de Yunguilla, coincidiendo con lo expuesto por Manuel Aragón (2014) y Lasso (2015), ambas buscan alternativas para continuar con el desarrollo de sus productos turísticos sostenibles.

Los proyectos creados con apoyo de la cooperación internacional y las ONG fueron diseñados para desarrollar la capacidad de manejo del turismo en áreas con alta biodiversidad a través del ecoturismo; generando capacidades para la captación de fondos y mantenimiento de los proyectos aún después de su finalización (Alex C. Walker Foundation et al., 2007).

Por otro lado, estos proyectos propendieron instituir en la población local la capacidad para instaurar un cambio estructural en la forma de percibir el desarrollo para mejorar su nivel de vida, lo que se refleja en la toma de decisiones, planificación y participación colectiva desde sus propias realidades, generando un crecimiento endógeno frente a los modelos de desarrollo externos (Boisier, 2001).

Los resultados identificados en este estudio no coinciden con el argumento de Orgaz-Agüera (2014) ya que, a pesar de la estacionalidad característica de la actividad turística, las dos comunidades han logrado generar recursos económicos. En el caso de Yunguilla, son reinvertidos en proyectos de desarrollo local, así como para la conservación de su patrimonio natural. Aunque no se puede mencionar que el caso de la comunidad de Oyacachi tenga el mismo éxito, han logrado combinar el turismo y la ganadería con un enfoque sostenible protegiendo el hábitat del oso andino en el Parque Nacional Cayambe Coca.

\section{Conclusiones}

Los resultados de este estudio determinan el éxito y el fracaso de la implementación de los proyectos de desarrollo del turismo sostenible en las dos comunidades objeto de este estudio, demostrando que la ayuda técnica y financiera de la cooperación internacional y las ONG con sus proyectos, logran establecer una base para el cambio del modelo productivo extractivista a un modelo sostenible en te- rritorios rurales con una importante biodiversidad y que lindan con la jurisdicción de áreas naturales protegidas.

La contribución de este estudio en el área del conocimiento es dejar de lado el estudio del turismo sostenible desde la perspectiva ambiental y económica, y obtener un análisis cualitativo sobre los alcances de esta modalidad de turismo enfocado en el pilar "social" del desarrollo sostenible, ya que identifica los alcances que han tenido los procesos implementados en las comunidades después de haber sido beneficiarias de los proyectos de cooperación.

Por lo dicho, se reconoce el caso exitoso de la comunidad de Yunguilla por su aplicación a varios fondos no reembolsables, mientras que en el caso de la comunidad de Oyacachi la capacidad para generar proyectos y continuar el desarrollo del turismo sostenible es nula.

La comunidad de Yunguilla forma parte de un proceso de cambio de un modelo extractivista a un modelo sostenible, gracias a la adopción de la conservación, el uso adecuado de sus recursos y la apuesta por el turismo desde 1995, después del impulso del proyecto de la ONG conservacionista. La comunidad de Yunguilla está constituida por un grupo de personas que, gracias a su organización comunitaria, sacan adelante proyectos alternativos para generar recursos económicos, con el fin de mejorar la calidad de vida de sus pobladores y la conservación de la biodiversidad y cultura en su territorio.

En el caso de la comunidad de Oyacachi, no se puede hablar de un desarrollo después de la asistencia de cooperación internacional y ONG desde la implementación de turismo sostenible, puesto que con el pasar del tiempo, la comunidad no se ha empoderado de los procesos implementados, y aún no se visibiliza un trabajo propio que impulse el desarrollo de alternativas productivas locales bajo su visión y capacidades que logre el desarrollo sostenible en Oyacachi.

Se concluye que el alcance del turismo sostenible, adoptado como un mecanismo de desarrollo en las dos comunidades rurales, depende de sus propias capacidades para la toma de decisiones, planificación y participación colectiva a partir de sus realidades y necesidades. Los resultados demuestran que la asistencia técnica y económica pueden ser las dos caras de una moneda; 
mientras que en una comunidad puede observarse un proceso sostenido de desarrollo, la otra aún no logra establecer un camino a su desarrollo.

Cabe resaltar que las limitaciones de este estudio están sujetas a los resultados de documentos técnicos sobre los procesos de ejecución de los proyectos de

\section{Referencias}

Acuerdo Ministerial No 063 del 2000 [Ministerio del Trabajo]. Aprobación del estatuto de la Corporación Microempresarial Yunguilla. 05 de junio de 2000.

Aigeneren, M. (1999). Análisis de contenido: Una introducción. La Sociología en sus Escenarios, 3, 1-52

Alex C. Walker Foundation, Nature Conservancy, US, \& United States, AID. (2007). Turismo, áreas protegidas y comunidades. Estudios de caso y lecciones aprendidas del Programa de Parques en Peligro 2002-2007. Región de Conservación de Sudamérica, Publicaciones Técnicas de Asuntos Externos No. 1. The Nature Conservancy.

Altés, C. (2006). El turismo en América Latina y el Caribe y la experiencia del BID. Banco Interamericano de Desarrollo (Sustainable Development Department Technical papers series; ENV-149).

Andréu Abela, J. (2000). Las técnicas de análisis de contenido: una revisión actualizada. Fundación Centro Estudios Andaluces - Universidad de Granada, 10(2), 1-34.

Arrueta, J. A. (1994). Ecoturismo en la reserva Cuyabeno: realidad y retórica en la relación de los Siona con el turismo ecológico. FLACSO Sede Ecuador.

Azócar de Buglass, L. (1995b). Ecoturismo, ¿Una alternativa de desarrollo sostenible?. En L. Azócar de Buglass (Comp.) Ecoturismo en el Ecuador: Trayectorias y desafios. (pp. 9-53). Cooperación para el Desarrollo y la Ayuda Humanitaria [DDA-Suiza), Intercooperation \& Oficina Regional para América del Sur de la Unión Mundial para la Naturaleza [UICN]. conservación, por lo que se recomienda la realización de un estudio a profundidad que evalúe los siguientes alcances: el equilibrio entre lo social y lo económico, la viabilidad entre lo ecológico y lo económico; lo equitativo entre lo económico y lo social, sobre los procesos de desarrollo sostenible adoptados en las dos comunidades objeto de este estudio.

Azócar de Buglass, L. (Comp.) (1995a). Ecoturismo en el Ecuador: Trayectorias y desafios. Cooperación para el Desarrollo y la Ayuda Humanitaria [DDA-Suiza), Intercooperation \& Oficina Regional para América del Sur de la Unión Mundial para la Naturaleza [UICN].

Bermeo Noboa, A. (2002). Desarrollo sustentable en la República del Ecuador. Dirección de Planificación del Ministerio del Ambiente (Ecuador).

Boisier, S. (2001). Desarrollo (local): ¿De qué estamos hablando? En O. Madoery, \& A. Vázquez Barquero (eds.), Transformaciones globales, Instituciones y Políticas de desarrollo local (pp. 1-22). Editorial Homo Sapiens.

Bustamante, M. (2007). Politica de reinversión para el complejo termal de la comunidad de Oyacachi en la Reserva Ecológica Cayambe Coca. Programa Parques en peligro. Ecociencia, Fundación Ecológica Rumicocha, Ministerio del Ambiente, Fundación Antisana, The Nature Conservancy, \& USAID.

Caiza, R., \& Molina, É. (2012). Análisis histórico de la evolución del turismo en territorio ecuatoriano. Revista Turismo, Desarrollo y Buen Vivir, 4, 6-24.

Cárdenas Toscano, M. T. (2016). Propuesta del plan estratégico comunicacional para el posicionamiento del turismo comunitario de la Corporación Yunguilla en la provincia de Pichincha. Universidad Central del Ecuador.

Carrera, M., Bustamante, M., \& Sáenz, M. (2016). Las áreas protegidas del Distrito Metropolitano de Quito: conocer nuestro patrimonio natural. SAMDMQ, Fondo Ambiental, CONDESAN, Proyecto EcoAndes-Programa Bosques Andinos. 
Carrillo García, M., Enríquez Rocha, P., \& Meléndez Herrada, A. (2017). Gestión comunitaria y potencial del aviturismo en el Centro de Ecoturismo Sustentable El Madresal, Chiapas, México. El Periplo Sustentable, 33, 564-604.

Carvalho, J., \& Rodríguez, A. (2006). Operadora de ecoturismo comunitario de Oyacachi. Plan de negocios. Ecociencia

Centro para la Investigación de la Diversidad Cultural y Biológica de los Bosques Pluviales Andinos [DIVA]. (2000). Oyacachi. La gente y la biodiversidad. Ediciones Abya-Yala.

Comité de Ecoturismo Oyacachi y EcoCiencia. (2007). Plan Estratégico 2004-2007, Planes de Acción 2004-2006. Programa Parques en Peligro.

Coppin, L. (1992). Ecoturismo y América latina: una aproximación al tema. Estudios y Perspectivas en Turismo, 1(1), 7-14.

Corporación Microempresarial Yunguilla. (2018). Plan de vida de las comunidades del área de conservación y uso sustentable Yunguilla Santa Lucía. Corporación Microempresarial Yunguilla.

Corporación Microempresarial Yunguilla. (2020). Historia de Yunguilla. Obtenido de www.yunguilla.org.eceshistoria

Cruz Albornoz, J. (1993). Situación actual del ecoturismo en Ecuador. Estudios y Perspectivas en Turismo, 2(4), 321-329.

Espín López, J. V. (2002). El análisis de contenido: una técnica para explorar y sistematizar información. XXI. Revista de Educación, 4, 95-105.

Flores, S. (2007). Informe final. Actividades. Implementación de un proyecto de ecoturismo en Oyacachi. Implementación de un plan de acción para el manejo del conflicto oso andino - ganado en Oyacachi. Programa Parques en peligro. Ecociencia, USAID, \& The Nature Conservancy.

Fondo para la protección del Agua [FONAG]. (2018). Levantamiento de información para la elaboración del guion cultural de la comunidad de Oyacachi. FONAG.
Hernández Pardo, P. (2011). Ecoturismo en Ecuador: El caso del Oglán alto. ECA Sinergia, 2(1), 9-17.

Instituto Nacional de Estadística y Censos del Ecuador. (2019). Población por parroquias del Ecuador. Ecuador en cifras. Obtenido de https:// www.ecuadorencifras.gob.ec/search/POBLACIÓN+POR+SEXO,+SEGÚN+PROVINCIA,+PARROQUIA+Y+CANTÓN+DE+EMPADRONAMIENTO/

Jara Chávez, H. (2006). Tulipe y la cultura Yumbo: arqueología comprensiva del subtrópico quiteño. Tomo I. Fondo de Salvamento del Patrimonio Cultural de Quito [FONSAL].

Jovanovic, R., Sánchez, D. C., Pavlovic, S., \& Devedzic, M. (2018). Principios de sostenibilidad en clusters de turismo rural: Los casos del Alto y el Bajo Danubio en Serbia. Revista de Geografia Norte Grande, 70, 211-233. https://doi. org/10.4067/S0718-34022018000200211

Kapera, I. (2018). Sustainable tourism development efforts by local governments in Poland. Sustainable Cities and Society, 40, 581-588. https:// doi.org/10.1016/j.scs.2018.05.001

Lasso, L. (2015). Ecoturismo en la Reserva Ecológica Cayambe Coca (RECAY): ¿mito u oportunidad?: El caso de la comuna kichwa de Oyacachi. Letras Verdes, Revista Latinoamericana de Estudios Socioambientales, 2, 17-18. https://doi.org/10.17141/letrasverdes.2.2008.814

Leal Londoño, M. del P. (2017). Turismo ecológico $y$ sostenible: Perfiles y tendencias. The Ostelea School of Tourism and Hospitality.

Ley de Gestión Ambiental del Ecuador. Ley Nro.9937, 38. Registro Oficial No. 245 14. 30 de julio de 1999.

López, A. (2005). Desarrollo sostenible: medio ambiente y turismo en las ciudades históricas: el caso de Toledo. Observatorio Medioambiental, 8, 331-344.

Manuel Aragón, M. (2014). Análisis del ecoturismo como alternativa de desarrollo sustentable en Latinoamérica (México, Costa Rica y Ecuador). El Colegio de la Frontera Sur. Université de Sherbrooke. 
Maquipucuna. (2019a). Yunguilla. Historia. Obtenido de http://www.yunguilla.org.ec/es/historia/

Maquipucuna. (2019b). Success Stories: Yunguilla. Obtenido de https://www.maquipucuna.org/ fundacion-maquipucuna?lang=es

Neudel, Y. (2015). La experiencia del turismo comunitario en Yunguilla, Ecuador y su impacto sociocultural en la comunidad. Letras Verdes. Revista Latinoamericana de Estudios Socioambientales, 18, 48-70. https://doi.org/10.17141/ letrasverdes.18.2015.1659

ONU. (2020). La Agenda para el Desarrollo Sostenible - Desarrollo sostenible. ¿Qué es el desarrollo sostenible? Obtenido de: https://www. un.org/sustainabledevelopment/es/development-agenda/

Orgaz-Agüera, F. (2014). Los impactos económi$\cos$, sociales y medioambientales negativos en el ecoturismo: Una revisión de la literatura. Nómadas. Critical Journal of Social and Juridical Sciences, 42(2), 139-148. http://dx.doi. org/10.5209/rev_NOMA.2014.v42.n2.48781

Orgaz-Agüera, F., \& Cañero Morales, P. (2015). Ecoturismo en comunidades rurales: análisis de los impactos negativos para la población local. Un estudio de caso. REVESCO. Revista de Estudios Cooperativos, 120, 99-120. https://doi. org/10.5209/rev_REVE.2016.v120.49155

Orgaz-Agüera, F., \& Cañero Morales, P. (2017). Ecoturismo y desarrollo sostenible. Un estudio de caso en comunidades rurales de República Dominicana. PASOS Revista de Turismo y Patrimonio Cultural, 13(6), 1425-1435. https:// doi.org/10.25145/j.pasos.2015.13.099

Pons, J. M., \& López, L. (2006). Turismo alternativo y reservas naturales. Cuadernos del Patrimonio Cultural y Turismo, 14, 151-165.

Prieto, M. (2011). Los estudios sobre turismo en Ecuador. En M. Prieto (ed.,) Espacios en disputa: el turismo en Ecuador (pp. 9-27). FLACSO, Sede Ecuador.

Reátegui Lozano, R. (2003). Fundamentos del desarrollo sostenible. Revista del Instituto de Investigación de la Facultad de Ingeniería Geológica, Minera, Metalúrgica y Geográfica,
6(12), 67-80. https://doi.org/10.15381/iigeo. v6i12.745

Rueda Flores, S. A. (2015). Análisis de la incidencia de la cooperación internacional en la comunidad de Yunguilla. Pontificia Universidad Católica del Ecuador.

Sánchez Valdés, A., \& Vargas Martínez, E. E. (2015). Turismo sustentable. Un acercamiento a su oferta. Multiciencias, 15(3),347-354.

Secretaría de Ambiente del GAD DMQ. (2015). Informe técnico sobre el informe final de acuerdo a la ejecución del proyecto del convenio firmado entre la SA-FA y la Corporación Yunguilla, para la "Ejecución del plan de manejo del ACUS Yunguilla (en aplicación de la OM 409 de 20 de junio de 2013)". Secretaría de Ambiente del GAD DMQ

Secretaría de Turismo [SECTUR]. (2004). Turismo alternativo. Una nueva forma de hacer turismo (Segunda Ed). Fascículo 1, Serie Turismo Alternativo. Secretaría de Turismo, Subsecretaría de Operación Turística, Dirección General de Desarrollo de Productos Turísticos, Dirección de Desarrollo de Turismo Alternativo.

Strydom, A. J., Mangope, D., \& Henama, U. S. (2019). A critique of the interface between tourism, sustainable development and sustainable tourism in communitybased tourism theory. African Journal of Hospitality, Tourism and Leisure, 8(5), 1-12.

Sundström, S. (2003). El ecoturismo como instrumento para desarrollo sostenible: Un estudio comparativo de campo entre Suecia y Ecuador. Institutionen för Kultur och kommunikation.

Torres Fernández, C., Zaldívar Martínez, P., \& Enríquez García, F. (2013). Turismo alternativo y educación: Una propuesta para contribuir al desarrollo humano. El Periplo Sustentable, 24, 125-154.

Vargas Martínez, E. E., Castillo Nechar, M., \& Zizumbo Villarreal, L. (2011). Turismo y sustentabilidad: Una reflexión epistemológica. Estudios y Perspectivas en Turismo, 20(3), 706-721. 
World Tourism Organization [UNWTO]. (2019). UNWTO World Tourism Barometer and Statistical Annex, May 2019. UNWTO World Tourism Barometer (English version), 17(2), 1-40. https://doi.org/10.18111/wtobarometereng.2019.17.1.2

World Tourism Organization [UNWTO]. (2020a).
Barómetro OMT del Turismo Mundial, mayo 2020 - Con especial enfoque en el impacto de la COVID-19 (resumen). UNWTO. https://doi. org/10.18111/9789284421831

World Tourism Organization [UNWTO]. (2020b). Turismo sostenible. https://www.unwto.org/es/ desarrollo-sostenible 